\title{
Long-term effects of lung cancer computed tomography screening on health-related quality of life: the NELSON trial
}

\author{
K.A.M. van den Bergh*, M.L. Essink-Bot*\#, G.J.J.M. Borsboom*, E.T. Scholten`, \\ R.J. van Klaveren ${ }^{+}$and H.J. de Koning*
}

ABSTRACT: The long-term effects of lung cancer computed tomography (CT) screening on health-related quality of life (HRQoL) have not yet been investigated.

In the Dutch-Belgian Randomised Lung Cancer Screening Trial (NELSON trial), 1,466 participants received questionnaires before randomisation (T0), 2 months after baseline screening (screen group only; T1) and at 2-yr follow-up (T2). HRQoL was measured as generic HRQoL (12-item shortform questionnaire and EuroQoL questionnaire), anxiety (Spielberger State-Trait Anxiety Inventory) and lung cancer-specific distress (impact of event scale (IES)). Repeated measures of ANOVA were used to analyse differences between the screen and control groups, and between indeterminate (requiring a follow-up $\mathrm{CT}$ ) and negative screening result groups.

At T0 and T2 there were no significant differences in HRQoL scores over time between the screen and control groups, or between the indeterminate or negative second-round screening result group. There was a temporary increase in IES scores after an indeterminate baseline result (T0: mean 4.0 (95\% Cl 2.8-5.3); T1: mean 7.8 (95\% Cl 6.5-9.0); T2: mean 4.5 (95\% Cl 3.3-5.8)).

At 2-yr follow-up, the HRQoL of screened subjects was similar to that of control subjects, the unfavourable short-term effects of an indeterminate baseline screening result had resolved and an indeterminate result at the second screening round had no impact on HRQoL.

KEYWORDS: Computed tomography, lung neoplasms, mass screening, quality of life

E ffective policy decisions regarding cancer screening programmes require data on the effects of screening on mortality, healthrelated quality of life (HRQoL) and their costeffectiveness [1]. Few studies have examined the HRQoL effects of lung cancer screening with computed tomography (CT) [2-5]. Subjects receiving an indeterminate or positive result for a baseline CT screening have reported increased anxiety or fear of cancer [2] and more lung cancerspecific distress than subjects with a negative result [3]. At short-term follow-up, when all subjects had negative CT results, these unfavourable effects on HRQoL were shown to have decreased and the differences between subjects with initially negative or positive/indeterminate results were no longer observed [2, 4, 5]. CT scanning caused only a little discomfort and had no major impact on HRQoL [4].

However, within a screened cohort, comparisons of HRQoL are of limited value due to the possible effects of reassurance and selection. The best method to evaluate the long-term impact of screening is to compare a group of screened participants with a control group in a randomised controlled trial (RCT) [1]. The rationale for this is, first, because a study population is often a selective group that is healthier than the general population $[6,7]$ and, secondly, because subjects invited for lung cancer screening differ from the general population in that they are usually heavy current or former smokers. The Dutch-Belgian Randomised Controlled Lung Cancer Screening Trial (NELSON trial) evaluates the long-term impact of screening on HRQoL [8].

An indeterminate result at baseline (i.e. first or prevalence screening) has an unfavourable effect on HRQoL, but it is unknown whether subjects experience a similar decrease in HRQoL after second-round screening (i.e. incidence screening).

The aims of the present study were to: 1) compare HRQoL in a screen and control group over 2 yrs;
AFFILIATIONS

*Depts of Public Health, +Pulmonology, Erasmus MC University Medical Centre, Rotterdam,

"Dept of Social Medicine, Academic Medical Centre, University of Amsterdam, Amsterdam, and "Dept of Radiology, Kennemer Gasthuis Haarlem, Haarlem, The Netherlands.

CORRESPONDENCE K.A.M. van den Bergh Dept of Public Health, Erasmus MC University Medical Centre Rotterdam P.O. Box 2040 3000 CA Rotterdam the Netherlands E-mail: k.vandenbergh@ erasmusmc.nl

Received: Aug 032010 Accepted after revision: Oct 302010 First published online: Dec 092010 
2) explore the short-term effects on HRQoL of an indeterminate result at second-round screening; 3) evaluate the long-term effects of an indeterminate baseline result; and 4) evaluate the differences between getting a negative follow-up scan and getting at least one indeterminate or positive result at followup.

\section{METHODS}

\section{NELSON study population}

The selection criteria for the NELSON trial have been described in detail previously [9]. Subjects randomised to the screen group could receive either a positive, indeterminate or negative test result at each screening round [10]. At baseline, a positive test was obtained when a nodule had a solid component that was $>500 \mathrm{~mm}^{3}$ (>9.8 $\mathrm{mm}$ in diameter) and required referral to a pulmonologist for work-up and diagnosis. An indeterminate result was obtained if the volume of the solid component or the solid component of a partially solid nodule was $50-500 \mathrm{~mm}^{3}$ (4.6-9.8 $\mathrm{mm}$ in diameter), or the diameter of a nonsolid nodule was $\geqslant 8 \mathrm{~mm}$. These participants were scheduled to undergo a follow-up CT scan to evaluate whether the lung nodule had grown (fig. 1). The follow-up period for an indeterminate result was 3 months after baseline screening. After the second screening round, the follow-up period was 6-8 weeks for subjects with new nodules and $1 \mathrm{yr}$ for subjects with previously existing nodules with a volume doubling time of 400-600 days [10]. Participants with an indeterminate test result received a letter stating that a very small abnormality had been found in the lung (5-10 $\mathrm{mm}$ in diameter), that this was a common finding that usually represents a small scar or minor inflammation only and that at this moment there was no need for any further investigations. Participants with a negative $\mathrm{CT}$ result at baseline or at second-round screening were invited to an annual repeat scan (second screening round) or a bi-annual scan (third screening round).

The entire NELSON trial, including this HRQoL study, was approved by the Dutch Ministry of Health and by the local ethics committees of the participating centres. Informed consent was obtained from all participants. The NELSON trial is registered at the Netherlands Trial Register (www.trialregister.nl).

\section{HRQoL study}

A sample of 1,466 subjects participating in the NELSON trial was taken before they were randomised (733 in both the screen group and the control group) (fig. 1). All participants received a questionnaire before trial randomisation (T0: baseline HRQoL assessment). A second questionnaire (T1) was sent 2 months after baseline screening to 684 screen participants with either a negative baseline result $(n=541)$ or an indeterminate baseline result $(n=143)$. The final questionnaire was sent at 2 -yr assessment (T2) to 682 screen participants and a random sample of 498 control participants out of 726 eligible participants; for the screen participants this was $\sim 1.5$ yrs after baseline screening, i.e. 0.5 yrs after the second-round screening. 51 (7.0\%) screen participants who did not undergo CT scans $(n=28)$ or who went "off screening" $(n=23)$, were not sent the T2 questionnaire. Reasons for being off screening were screen-detected lung cancer $(n=9)$, no longer wishing to participate $(n=10)$, died $(n=2)$, or could not be contacted $(n=2)$. In addition, seven out of the 733 control group participants were not eligible for the T2 questionnaire because they had died $(n=4)$ or no longer wished to participate in the trial $(n=3)$.

\section{Measures}

Generic HRQoL

The participant's generic HRQoL was measured with the 12-item short-form (SF-12) questionnaire and the EuroQol questionnaire (EQ-5D) [11-14]. The SF-12 is a shorter version of the 36-item short-form questionnaire and consists of a physical component summary (PCS) and a mental component summary (MCS) [14]. We used the acute (1-week recall) form of version 1, in which a higher score indicates a better HRQoL. Respondents were also asked to rate their own health on the visual analogue scale (VAS) of the EQ-5D, ranging from 0 (worst imaginable health status) to 100 (best imaginable health status) $[11,13]$.

\section{Generic anxiety}

Generic anxiety was measured using the short form of the Spielberger State-Trait Anxiety Inventory (STAI-6) [15]. Six items related to anxiety (calm, tense, upset, relaxed, content and worried) were rated on a four-point scale. The total summary score was calculated in subjects with a maximum of three missing values and could range from 20 to 80 , with higher scores indicating more anxiety [16]. The STAI-6 is reported to have good reliability and validity, and was found useful in evaluating the effectiveness of screening programmes on subjective anxiety levels [15].

\section{Lung cancer-specific distress}

Lung cancer-specific distress was measured using the impact of event scale (IES) [17, 18]. The 15 IES items were tailored to lung cancer as the specific stressor. Each item was scored on a four-point scale: not at all (score of 0 ); rarely (score of 1 ); sometimes (score of 3); and often (score of 5). The total score and subscales (avoidance and intrusion) were calculated for those who completed $75 \%$ of the questions on each subscale, and were corrected for the total number of questions on the subscale. The total summary score could range 0-75 (intrusive scale 0-35, avoidance scale 0-40), with a higher score indicating more lung cancer-specific distress.

\section{Demographic and other data}

At T0, marital and smoking status was measured. Educational level and smoking pack-yrs were derived from the first NELSON questionnaire that was used for selection of the NELSON trial participants [9].

\section{Statistical analysis}

Differences in respondent characteristics between the screen and control groups were analysed using Chi-squared tests and Mann-Whitney U-tests because these data were not normally distributed.

Analysis of each research question required different datasets. For the first research question, data of participants in the NELSON trial who received both $\mathrm{T} 0$ and $\mathrm{T} 2$ questionnaires and returned at least one questionnaire were used (screen arm: $n=665$; control arm: $n=460$ ). For the second question, screen group participants were included who received both T0 and T2 questionnaires, returned at least one questionnaire and had an indeterminate $(n=49)$ or a negative $(n=585)$ result at the 


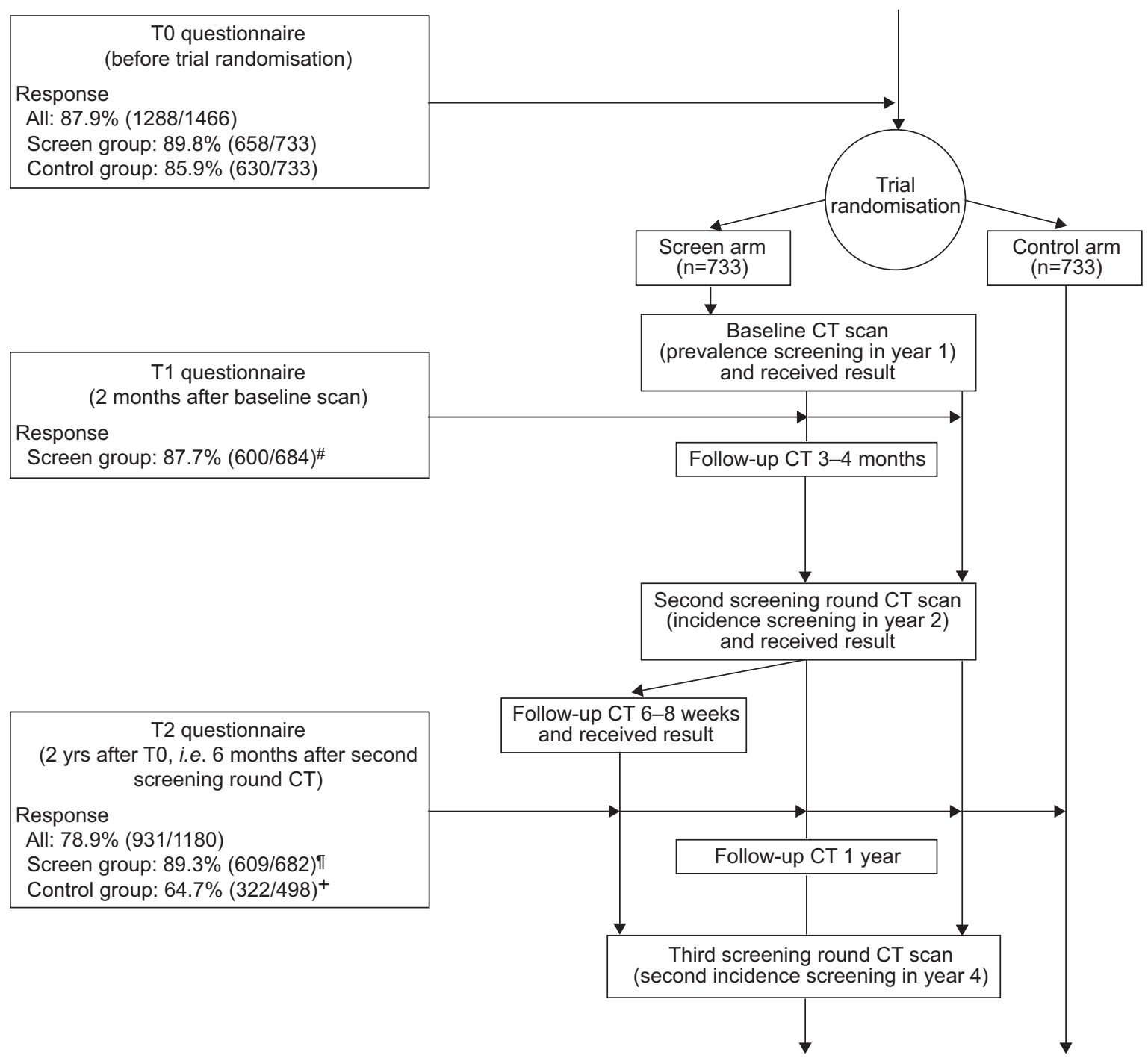

FIGURE 1. Flowchart of the health-related quality of life study in the screen and control groups. CT: computed tomography. ${ }^{*}$ : the questionnaire was sent to participants with either a negative $(n=541)$ or an indeterminate $(n=143)$ baseline scan result. $\%: 51(7.0 \%)$ screen group participants who did not undergo $C T$ scans $(n=28)$ for various reasons, or who were "off screening" ( $n=23)$ were not sent the T2 questionnaire. ${ }^{+}$: the T2 questionnaire was sent to a random sample of 498 control group participants out of 726 eligible participants, seven participants were not eligible for the T2 questionnaire.

second screening round. For the third question, data were used from the screen group participants who received T0, T1 and T2 questionnaires, who returned at least one questionnaire and who at baseline screening had either a negative result $(n=521)$ or an indeterminate result $(n=135)$. Furthermore, for the subgroup analyses of the latter group, subjects with at least one indeterminate $(n=28)$ or positive result $(n=7)$ and subjects with only negative results $(n=100)$ were analysed.

HRQoL changes over time and between groups

To analyse the HRQoL changes over time and differences between groups, random effects ANOVA was used to account for the repeated measurement of each subject. The "proc mixed" procedure in the SAS system version 9.1 (SAS Institute Inc., Cary, NC, USA) was used, allowing the use of all available data, including incomplete records. The models included a main effect for time, and the interaction between group and time. This parameterisation involved that the separate components in the interaction term tested the group differences at the consecutive measurement moments.

The following fixed covariates were added to the model: age [2, 19], sex [19], education [2], smoking status [20] and smoking pack-yrs (because we expected subjects with more pack-yrs to be more anxious and to have worse health). If the interaction term was not significant, models with the main effects for time and group were used, adjusted for covariates.

The IES scores were highly skewed. We considered repeated measures of ANOVA to be appropriate for the IES scores because a logistic regression model using a generalised linear mixed models approach analysis: 1 ) would reduce information content of the data, 2) has a cut-off point that is arbitrarily chosen, and 3) provides results that are comparable to those of repeated measures of ANOVA. 
A p-value $<0.05$ was considered statistically significant. To determine a clinically relevant difference between means at two assessment points or between the two subgroups, the minimal important difference (MID) was used. This is defined as half an SD. The MID can serve as a default value for important patient-perceived changes in HRQoL [21, 22].

\section{RESULTS}

The questionnaire response at T0 was $89.8 \%$ in the screen group and $85.9 \%$ in the control group; at T1 it was $87.7 \%$ (screen group only) and at T2 it was $89.3 \%$ in the screen group and $64.7 \%$ in the control group (fig. 1). Screen group participants completed T0 $4.9 \pm 3.6$ months before baseline screening, T1 at $1.3 \pm 0.8$ months after the baseline result and T2 $5.6 \pm 1.2$ months after the second screening round. For the screen and control group together, the time interval between $\mathrm{T} 0$ and T2 was $23.3 \pm 3.7$ months. No significant differences in sex, age, education and smoking characteristics were found between responders in the screen $(n=665)$ and control groups $(\mathrm{n}=460)$ (table 1).

HRQoL differences between the screen and control groups No statistically significant differences were found in HRQoL scores over time between the screen and control groups (table 2; see Appendices 1 and 2 in the online supplementary data). None of the parameters for time or trial arm, or the interaction between time $\times$ trial arm was significant for any of the HRQoL outcome measures.

\section{Short-term impact of an indeterminate second screening round result}

No statistically significant differences were found in HRQoL scores from baseline to 6 months after the second-round screening between subjects with an indeterminate or negative secondround screening result (table 2; Appendices 1 and 2 in the online supplementary data). None of the parameters for time or result, or the interaction between time $\times$ result, were significant for any of the HRQoL outcome measures.

\section{Long-term HRQoL differences between indeterminate and negative baseline results}

Subjects with a negative $(n=521)$ or indeterminate test result $(n=135)$ at baseline had received $1.0 \pm 0.3$ and $2.0 \pm 0.4$ new CT scans with results, respectively, when they had completed the T2 questionnaire.

For the group with a negative result at baseline, the results of the last follow-up CT at T2 were: $0.2 \%(n=1)$ positive, $6.5 \%(n=34)$ indeterminate and $93.3 \%(n=486)$ negative. For the group with an indeterminate result at baseline, the results of the last followup CT at T2 were indeterminate for $15(11.1 \%)$ subjects and negative for $120(88.9 \%)$ subjects.

The course of IES (total, intrusive and avoidance) scores over time differed between the groups with a negative and an indeterminate baseline result $(\mathrm{p}<0.01$ interaction time $\times$ result for all) (table 2 and fig. 2; Appendices 1 and 2 in the online supplementary data). In the indeterminate group the total IES scores changed from 4.0 (95\% CI 2.8-5.3), to 7.8 (6.5-9.0) and to 4.5 (3.3-5.8), whereas the negative group changed from 4.1 (3.4-4.8), to $2.6(2.0-3.3)$ and to $3.5(2.9-4.2)$ at T0, T1 and T2, respectively (Appendix 2 in the online supplementary data). No statistically significant differences in IES between result groups were found at $\mathrm{T} 0$ or $\mathrm{T} 2$. The course of the EQ-5D VAS and the MCS scores did not differ between the two result groups over time, but the group as a whole had worse scores at T1 compared with T2 (-1.3 and $-0.8, \mathrm{p}<0.01$ and $\mathrm{p}<0.05$, respectively) (table 2). Although PCS scores did not change over time, the indeterminate result group had worse PCS scores than the negative result group $(-1.4, p=0.04)$ (table 2). For all these analyses, only the differences between the result groups of the IES scores exceeded the MID at T1 and, therefore, only these were clinically relevant.

TABLE 1 Baseline characteristics of the screen and control group responders included in the health-related quality of life study of the Dutch-Belgian Randomised Controlled Lung Cancer Screening Trial (NELSON trial)

\begin{tabular}{|c|c|c|}
\hline & Screen group $\#$ & Control group \\
\hline Subjects $\mathbf{n}$ & 665 & 460 \\
\hline Age yrs & $57.8 \pm 5.5$ & $57.8 \pm 5.7$ \\
\hline \multicolumn{3}{|l|}{ Education } \\
\hline Primary education & 9.8 & 11.5 \\
\hline Higher vocational education or university education & 27.1 & 27.6 \\
\hline \multicolumn{3}{|l|}{ Smoking } \\
\hline Current smokers & 53.7 & 52.4 \\
\hline Pack-yrs & $39.7 \pm 17.4$ & $39.4 \pm 16.2$ \\
\hline
\end{tabular}




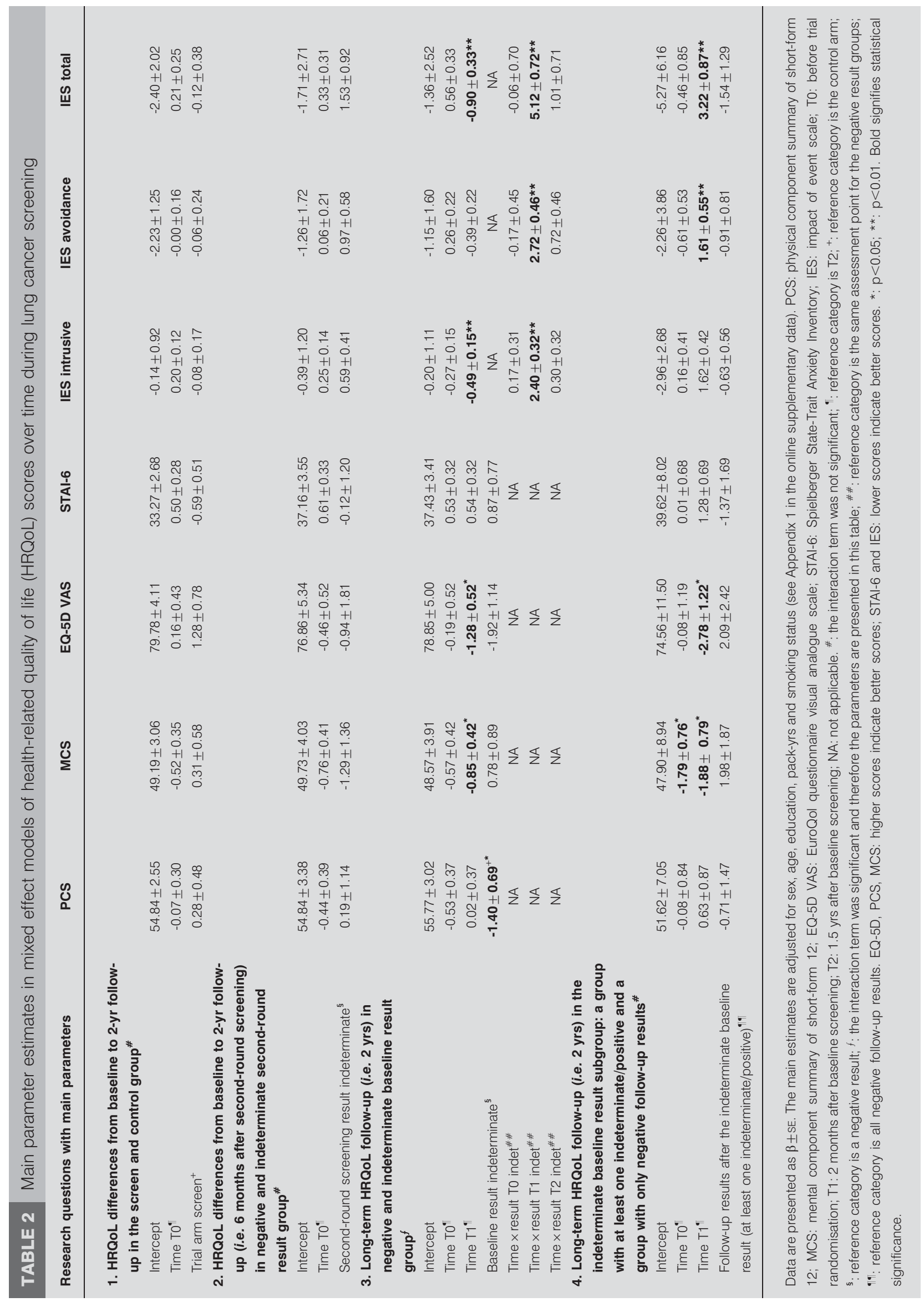



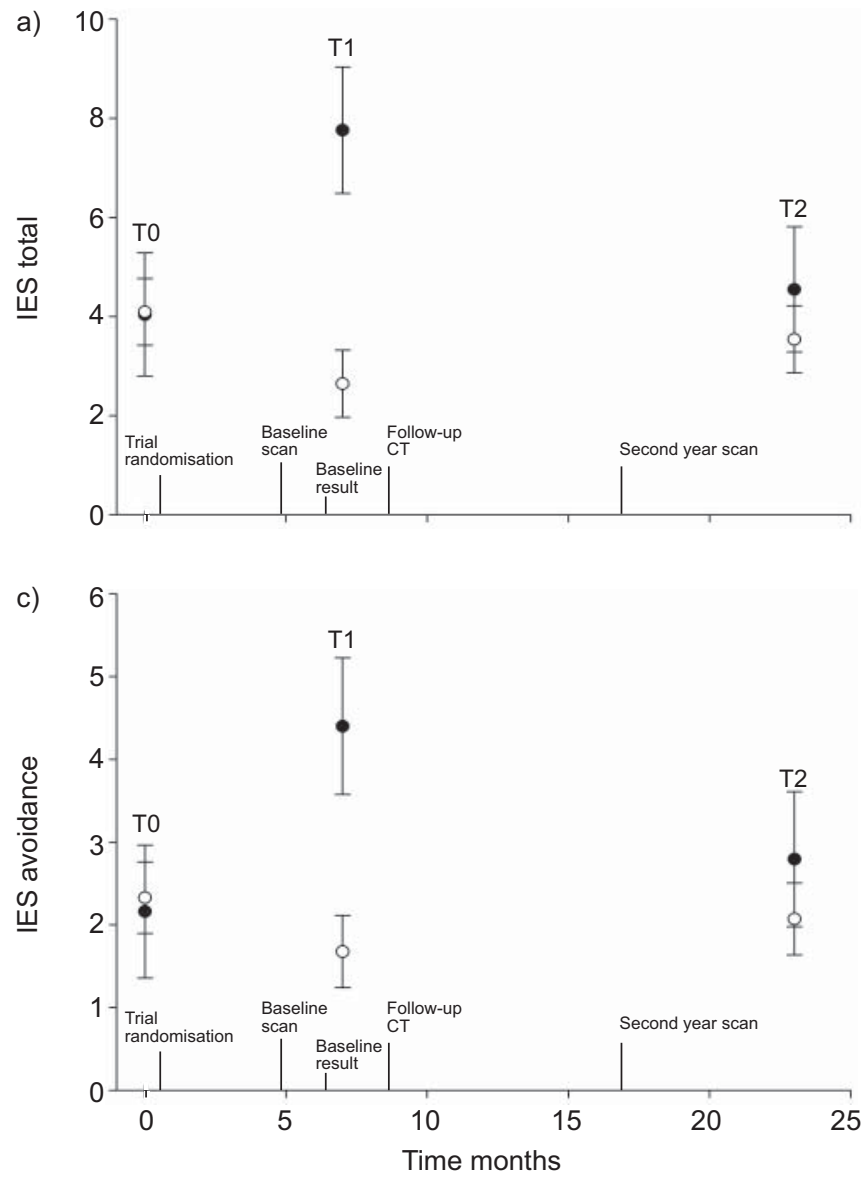

In the subgroup analyses of the indeterminate baseline result group (i.e. subgroups with subjects with at least one indeterminate/positive result at follow-up $(n=35)$ and subjects with only negative results at follow-up $(n=100))$, no statistically significant differences in HRQoL were found between the groups (table 2; Appendix 1 in the online supplementary data). The EQ-5D VAS and IES total, intrusive and avoidance scores were worse at T1 compared with T2 $(-2.8,1.6,1.6$ and 3.2, respectively; $\mathrm{p}=0.02, \mathrm{p}<0.01, \mathrm{p}<0.01$ and $\mathrm{p}<0.01$, respectively) and the MCS scores were also better at T2 compared with T0 $(-1.8)$ and T1 $(-1.9$; both $\mathrm{p}<0.02)$. None of these statistically significant differences exceeded the MID.

\section{Impact of covariates on HRQoL}

In general, the HRQoL scores were worse for females than for males ( $<<0.05$; Appendix 1 in the online supplementary data). Subjects with more pack-yrs and/or current smokers had statistically significantly worse self-reported health (EQ-5D VAS) and had statistically significant worse physical health scores (PCS) than subjects with less pack-yrs and former smokers $(p<0.05)$. Smoking status and pack-yrs were not associated with MCS and STAI-6. Current smoking status was negatively associated with IES $(p<0.01)$.

\section{DISCUSSION}

Within a randomised design, the present study showed no longterm negative effects of lung cancer screening on HRQoL at 2-yr follow-up. First, HRQoL was the same in both the screen group

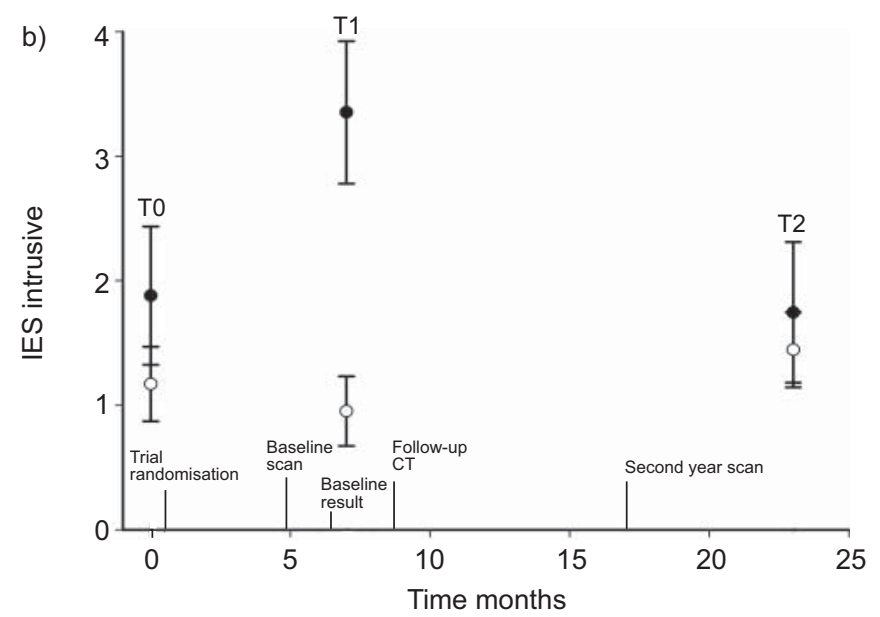

FIGURE 2. Average lung cancer-specific distress scale scores (where 0 is better and 10 is worse) of a) impact of event scale (IES) total, b) IES intrusive and c) IES avoidance. The scores were adjusted for sex, age, education, smoking status and smoking pack-yrs. Data are presented as least squares mean, horizontal lines indicate the 95\% confidence intervals. $\bigcirc$ : negative baseline result; $\bullet$ : indeterminate baseline result. T0: before trial randomisation; T1: 2 months after baseline screening; T2: 2-yr follow-up. CT: computed tomography.

and control group before trial randomisation and at 2-yr followup. Secondly, HRQoL was the same in subjects with an indeterminate and a negative second screening round result, both before randomisation and 6 months after the second screening. Thirdly, the negative effects on HRQoL after an indeterminate baseline result did not persist long term, even if an indeterminate baseline test result was followed by one or more positive or indeterminate test results.

Evaluating the HRQoL effects of lung cancer screening is ideal when including a randomised comparison of changes in HRQoL between those who underwent screening and those who did not. As far as we know, no other cancer screening studies have investigated HRQoL using a randomised screening design. Only TAYLOR et al. [19], in their prostate, lung, colorectal and ovarian cancer screening trial, reported baseline and 1-yr HRQoL average scale scores of the SF-12 in a screen and control group. In their study, according to the MID, no clinically relevant differences existed between the screen and control arm, and their scores were similar to our scores.

Until now, an unfavourable HRQoL effect of lung cancer screening was only found after an indeterminate or positive baseline scan result $[2,3,5]$. We found no unfavourable HRQoL effects 6 months after an indeterminate second screening round result requiring a 1-yr follow-up CT. This is remarkable because subjects with an indeterminate secondround screening received a result letter similar to that received after baseline screening. An explanation may be that many 
subjects become accustomed to such a result. $\sim 40 \%$ of the subjects had already received an indeterminate baseline result. Participants may also become reassured after a follow-up period of 1 yr. However, several participants and general practitioners telephoned the research centres after receiving the 1-yr follow-up recommendation. Therefore, a more plausible explanation for this finding is the timing of the HRQOL measurement after the second-round screening (i.e. 6 months after screening, which was also 6 months before the follow-up scan). A temporary negative impact on HRQoL may have occurred just after receiving the result of the secondround screening and/or just before the extra follow-up screening; however, this was not specifically assessed in our study. Nevertheless, if HRQoL was negatively affected by the indeterminate second-round screening result, this effect diminished over time since no unfavourable effect was found after 6 months.

Females showed a worse HRQoL than males. BYRNE et al. [2] also found significantly more anxiety in females than in males in a lung cancer-screening trial and TAYLOR et al. [19] found higher IES intrusive scores and MCS scores in females than in males in their screening trial. Similar to BYRNE et al. [2], who found a higher fear of cancer in current smokers, we found more lung cancer-specific distress in current smokers.

\section{Study limitations}

Differences in response rates between the screen and control groups may limit the validity of comparisons because of possible selection bias. The response rate at T2 was $89 \%$ in the screen group and $65 \%$ in the control group. However, the lower rate in the control group was estimated too low. We had less opportunity to correct the denominator in the control group for changes in address and serious events associated with being off screen. Furthermore, because comparison of respondents and non-respondents in the control group showed no significant differences in demographic characteristics, there is no evidence for selective response in the control group.

\section{Implications}

The only negative effect on HRQoL of lung cancer screening was a temporary increase in lung cancer-specific distress scores after an indeterminate baseline result; this does not seem to be an obstacle to the introduction of a lung cancerscreening programme.

\section{Conclusions}

In our study, lung cancer screening had no negative impact on HRQoL in the long term. At 1.5 yrs after baseline screening, subjects who did not have (screen detected) lung cancer had a HRQoL similar to that in control subjects, the negative shortterm effects of an indeterminate baseline screening result were resolved and an indeterminate second-round screening result had no negative impact on HRQoL 6 months later.

\section{SUPPORT STATEMENT}

This study was supported by the Netherlands Organisation for Health Research and Development (ZonMw; grant numbers 22000130 and 120610015), the Dutch Cancer Society (KWF; grant number EMCR 20012371) and the Health Insurance Innovation Foundation (Innovatiefonds Zorgverzekeraars). The funding sources had no involvement in our work.

\section{CLINICAL TRIAL}

The NELSON trial is registered at www.trialregister.nl (identifier number ISRCTN63545820).

\section{STATEMENT OF INTEREST}

None declared.

\section{ACKNOWLEDGEMENTS}

We would like to thank R. Faber (Dept of Public Health, Erasmus MC University Medical Centre, Rotterdam, the Netherlands) for establishing the questionnaire database and selecting the participants, A.C. de Jongh (Artex BV, Capelle aan den Ijssel, the Netherlands) for assistance with the selection of participants and handling of the mailings, M.A. Quak, for sending the questionnaires to the participants and F.J.P. Santegoets (both Dept of Public Health, Erasmus MC University Medical Centre) for assistance in linking the databases to add in the baseline scan results.

\section{REFERENCES}

1 Miller AB, Madalinska JB, Church T, et al. Health-related quality of life and cost-effectiveness studies in the European randomised study of screening for prostate cancer and the US Prostate, Lung, Colon and Ovary trial. Eur J Cancer 2001; 37: 2154-2160.

2 Byrne MM, Weissfeld J, Roberts MS. Anxiety, fear of cancer, and perceived risk of cancer following lung cancer screening. Med Decis Making 2008; 28: 917-925.

3 van den Bergh KA, Essink-Bot ML, Borsboom GJ, et al. Short-term health-related quality of life consequences in a lung cancer CT screening trial (NELSON). Br J Cancer 2010; 102: 27-34.

4 van den Bergh KA, Essink-Bot ML, Bunge EM, et al. Impact of computed tomography screening for lung cancer on participants in a randomized controlled trial (NELSON trial). Cancer 2008; 113: 396-404.

5 Vierikko T, Kivistö S, Järvenpää R, et al. Psychological impact of computed tomography screening for lung cancer and occupational pulmonary disease among asbestos-exposed workers. Eur J Cancer Prev 2009; 18: 203-206.

6 Otto SJ, Schroder FH, de Koning HJ. Low all-cause mortality in the volunteer-based Rotterdam section of the European randomised study of screening for prostate cancer: self-selection bias? J Med Screen 2004; 11: 89-92.

7 Zeliadt SB, Etzioni R, Ramsey SD, et al. Trends in treatment costs for localized prostate cancer: the healthy screenee effect. Med Care 2007; 45: 154-159.

8 van Klaveren RJ, Oudkerk M, Prokop M, et al. Management of lung nodules detected by volume CT scanning. N Engl J Med 2009; 361: 2221-2229.

9 van Iersel CA, de Koning HJ, Draisma G, et al. Risk-based selection from the general population in a screening trial: selection criteria, recruitment and power for the Dutch-Belgian randomised lung cancer multi-slice CT screening trial (NELSON). Int J Cancer 2007; 120: 868-874.

$10 \mathrm{Xu} \mathrm{DM}$, Gietema $\mathrm{H}$, de Koning $\mathrm{H}$, et al. Nodule management protocol of the NELSON randomised lung cancer screening trial. Lung cancer 2006; 54: 177-184.

11 Essink-Bot ML, Stouthard ME, Bonsel GJ. Generalizability of valuations on health states collected with the EuroQolc-questionnaire. Health Econ 1993; 2: 237-246.

12 Gandek B, Ware JE, Aaronson NK, et al. Cross-validation of item selection and scoring for the SF-12 Health Survey in nine countries: results from the IQOLA Project. International Quality of Life Assessment. J Clin Epidemiol 1998; 51: 1171-1178.

13 Kind P, Brooks R, Rabin R, eds. EQ-5D Concepts and Methods: A Development History. Dordrecht, Springer, 2005. 
14 Ware J Jr, Kosinski M, Keller SD. A 12-item short-form health survey: construction of scales and preliminary tests of reliability and validity. Med Care 1996; 34: 220-233.

15 van der Bij AK, de Weerd S, Cikot RJ, et al. Validation of the Dutch short form of the state scale of the Spielberger State-Trait Anxiety Inventory: considerations for usage in screening outcomes. Community Genet 2003; 6: 84-87.

16 Maissi E, Marteau TM, Hankins M, et al. The psychological impact of human papillomavirus testing in women with borderline or mildly dyskaryotic cervical smear test results: 6-month follow-up. Br J Cancer 2005; 92: 990-994.

17 Brom D, Kleber RJ. De Schok Verwerkings Lijst [The Dutch version of the Impact of Event Scale]. Ned Tijdschr Psychol 1985; 40: 164-168.
18 Horowitz M, Wilner N, Alvarez W. Impact of event scale: a measure of subjective stress. Psychosom Med 1979; 41: 209-218.

19 Taylor KL, Shelby R, Gelmann E, et al. Quality of life and trial adherence among participants in the prostate, lung, colorectal, and ovarian cancer screening trial. J Natl Cancer Inst 2004; 96: 1083-1094.

20 Laaksonen M, Rahkonen O, Martikainen P, et al. Smoking and SF36 health functioning. Prev Med 2006; 42: 206-209.

21 Norman GR, Sloan JA, Wyrwich KW. Interpretation of changes in health-related quality of life: the remarkable universality of half a standard deviation. Med Care 2003; 41: 582-592.

22 Turner D, Schunemann HJ, Griffith LE, et al. The minimal detectable change cannot reliably replace the minimal important difference. J Clin Epidemiol, 63: 28-36. 